\title{
Oocyte collection techniques in the dromedary camel
}

\author{
Tinson $^{1}$ A. H., A. O. McKinnon ${ }^{2}$, K. Singh ${ }^{1}$, K. S. Kuhad ${ }^{1}$ and R. Sambyal ${ }^{1}$ \\ ${ }^{1}$ Embryo Transfer Research Center for Racing Camels, Private Department of H. H. \\ The Crown Prince Sheikh Khalifa Bin Zayed Al Nahyan, P.O. Box 17292 Al-Ain \\ United Arab Emirates \\ ${ }^{2}$ Goulbourn Valley Equine Hospital, Numurkah, Australia
}

\begin{abstract}
The camel presents many challenges when it comes to trying to improve its breeding potential. Ultrasonic monitoring allows for more accurate prediction of the optimum time of mating as well as helping to manage camels being used for embryo transfer. However there are some camels that repeatedly fail to fall pregnant via natural means or using surrogates with embryo transfer. This can occur due to a variety of reasons, the most common of which is due to the presence of excessive amounts of fluid in the ovarian bursa of the dromedary camel. I.V.F. technology, now in everyday use in humans facing fertility problems, has been looked at experimentally as a possible way to solve this. Collection of the oocyte is obviously central to the process and in this paper we compare three routes of collection, discuss problems encountered in the procedures and the likely future of experiments with this technology.
\end{abstract}

Key words: oocyte, ultrasound, embryo transfer, camel.

\section{Introduction}

Camel racing is a highly lucrative and well-organized sport in this part of the world. It has become highly competitive with the offer of prizes such as luxurious cars, gold cups and prize money. The support of the industry has seen it evolve over the last $20 \mathrm{yrs}$ from an essentially traditional sport to a sophisticated industry. Racing camels are now finely tuned athletes with computer chip identification, strict diets and stringent training programmes. The racing camels are categorized in different groups according to their age, sex and breed and only allowed to compete within that group. In camel racing, female has an upper hand to the male (Tinson et al., 1998). The rewards for the local trainers to train a champion race camel are considerable. The racing life of a female camel is considerable (Manefield and Tinson 1996) and having retired from racing the importance of producing offspring becomes paramount. The low calving rates associated with traditional camel breeding (Wilson 1984) have led to the application of assisted reproductive technology to camel breeding to assist (McKinnon et al., 1994; Tibary and Anouassi 1997). While embryo transfer has greatly helped to speed the breeding of the camel and help solve some infertility problems (Tinson et al., 2001) there are still cases of infertility that have eluded successful treatment. Certain conditions such as ovarian bursal fluid syndrome, blockage of the oviduct (hydrosalpinx, pyosalpinx and mucosal cysts) and congenital or acquired abnormalities of the uterus and or cervix (segmental aplasia, uterine cysts, remnants of mesonephric and paramesonephric ducts as well as parturition trauma), (Tibary and Anouassi 1997) can lead to reproductive failure. In such conditions when both natural and $\mathrm{E} \mathrm{T}$ breeding programs are unsuccessful then Assisted/Advanced Reproductive Techniques (ART) are the only way to achieve the desire goal. These technologies include, In Vitro Fertilization (IVF), Gamete Intrafallopian Transfer (GIFT), Zygote Intrafallopian Transfer (ZIFT) and Intra Cytoplasmic Sperm Injection (ICSI). In Vitro Fertilization Process involves retrieving eggs (oocytes) and sperm from female and male and placing them in together in laboratory dish for fertilization and transferred back to the female reproductive tract for implantation and subsequent development. In humans and other domesticated animals such as cattle, 
sheep many of these similar problems led to the successful application of IVF to routinely aquire successful pregnancy. The technique was first pioneered in rabbits with IVF offspring births in 1959. The first human birth was that of Louise Brown in 1978 and the birth of large domestic IVF offspring following in 1981 with the first live calf. In some species such as the horse, the technique has been difficult with reproducible results not occurring till as recent as 1998 when I.C.S.I was applied to solve the problem (McKinnon et al., 1988). In the camel the high incidence of ovarian bursal fluid syndrome in referred cases of infertility to our center $(30 \%$ of infertile camels with no offspring in over 3yrs) and the high dollar value of the camels involved make success with the techniques a major reproductive objective for the future. However before it will be possible to see success in the techniques a reproducible and safe technique of oocyte collection must be developed for the racing camel. Ultrasound guided collection via a transvaginal probe developed as method of collection in the Netherlands in the late 80's and soon was established as a safe and repeatable technique in the horse and cow (Carnevale and Ginther, 1993. Meinijetes et al. 1995). It is even being used in the first trimester of pregnant cattle. Once genetic material can be obtained from problem camels on a regular basis with ease then the laboratory work can be built up to attain successful fertilisation and hopefully transferable embryos.

\section{Materials and Methods}

\section{Selection and preparation of Donors}

Nineteen female camels were selected on the basis of a normal breeding history and the absence of any abnormalities in the reproductive tract based on an ultrasonic examination (Mc Kinnon and Tinson 1992; Tibary and Anouassi 1997). The camels were separated into groups of 3 animals and a regime of 14 days of progesterone injections intramuscularly, daily (100mg Bomagest NZ) followed by a superovulatory regime of FSH over 7 days. The techniques of batching the groups and synchronising the animals have been previously described in other work (McKinnon et al., 1994. Tinson et al., 1998). On $9^{\text {th }}$ day donors were scanned for the superovulatory response (Mc Kinnon and Tinson 1992) and 3000 IU of Human Chorionic Gonadotrophin (HCG) (Chorulon Intervet Neth.) was given to facilitate the maturation according to the time of collection (at $20 \mathrm{hr}, 24 \mathrm{hr}$ and $>24 \mathrm{hr}$ ).

\section{Collection of oocytes}

According to the collection techniques the donors were categorized into three groups and designated $\mathrm{A}, \mathrm{B}$ or $\mathrm{C}$.

- Group (A) consisted of 13 camels which were collected on 5 consecutive days via a non surgical transvaginal technique.

- Group (B) consisted of 3 camels and had the left ovary collected surgically via laparotomy with the right ovary receiving no treatment.

- Group (C) also consisted of 3 camels and had the left ovary collected via laparotomy and the right ovary collected via transvaginal technique. Group B and $\mathrm{C}$ were grouped together and done on 4 consecutive days.

\section{Surgical Technique}

Donors were prepared for aseptic standing flank laparotomy under sedation with an intravenous injection of $.75 \mathrm{cc}$ of Ketamine and $.75 \mathrm{cc}$ of Xylazine (Ketamine hydrochloride $100 \mathrm{mg} / \mathrm{ml}$ and Xylazine hydrochloride $100 \mathrm{mg} / \mathrm{ml}$, Parnell, Australia). A vertical line of $20 \mathrm{~cm}$ was infiltrated with $80 \mathrm{ml}$ of local anaesthesia (prilocaine hydrochloride $20 \mathrm{mg} / \mathrm{ml}$, Parnell Aust) on the left flank of the abdomen of the camel approximately $10 \mathrm{~cm}$ infront of the anterior crest of the ilium. The surgery 
site was shaved and prepared for sterile surgery with a surgical drape placed over the surgical site. Using sterile gloves and instruments the skin and muscle were cut open to gain access to the peritoneal cavity and the left ovary. After exteriorization of left ovary through incision line individual follicle were aspirated by 18 Gauge needle attached to a 20cc syringe. The syringe had previously been filled with $1 \mathrm{cc}$ of a gamete wash solution mixed with heparin (Minihep 25,000IU/ml heparin Leo Labs Holland) at a concentration of 25 I.U. heparin $/ \mathrm{ml}$ of wash solution (Bovine Vitro Wash V-BVWA-50, Cook Australia). The aspirated follicle fluid was then put into holding tubes of $15 \mathrm{cc}$ capacity, which already had $1 \mathrm{cc}$ of the heparin/wash mix placed inside. The tubes were held at $37 \operatorname{deg} \mathrm{C}$ by way of a Tube Heater (VFTH-1000, Cook Australia) till all the follicles were aspirated and the fluid could be readied for examination under the microscope. Once the aspiration was complete the abdominal muscles were sutured together with Catgut (USP 3, Kruuse Germany) using a continuous horizontal mattress suture then a simple continuous suture subcutaneously with Silk USP 3 (Kruuse Germany) in a simple interrupted mattress stitch for the skin.

Non Surgical Technique/ Transvaginal ultrasound guided oocyte aspiration

Donors were sedated and prepared by using $.5 \mathrm{cc}$ of both Ketamine and Xylaxine given intravenously with an epidural injection (Manefield and Tinson 1996) of $5 \mathrm{cc}$ of Prilocaine. The perineum was washed with a Hibicet solution (Chlorhexidine gluconate1.5\% w/v and cetrimide $15 \% \mathrm{w} / \mathrm{v}$, Zeneca UK) then with water followed by a dry Chux towel (Johnson and Johnson Aust). An Aloka ultrasound unit (SSD-500 Aloka Japan) with $5 \mathrm{MHz}$ convex transvaginal probe mounted with a needle guide (UST-994p5 Aloka) was placed in position behind the camel along with a regulated vacuum pump designed for oocytre aspiration (VMAR-5000, Cook Aust) and the tube heating set (VFTH-1000, Cook). Four $15 \mathrm{cc}$ caped tubes were placed in the positions in the heater, all having previously received $1 \mathrm{cc}$ of heparin/wash solution $(25 \mathrm{IU} / \mathrm{ml})$. A bovine aspiration set was then attached to the vacuum unit and to the first of the tubes. The needle used was a single lumen $60 \mathrm{~cm}, 17$ guage needle (V-BOAS-1760-S, Cook Aust) with a small silicon stopper suitable to seal the $15 \mathrm{cc}$ tubes top. When the operator is ready and the sedation is deemed to have taken effect the probe end is smeared with $\mathrm{K}-\mathrm{Y}$ jelly (Johnson Johnson Aust) and a plastic rectal globe is applied over the head of the probe so as to cover the entire probe length and keep the plastic, jelly and probe end in close contact. The probe is then introduced into the vagina to the fornix of the canal and angle to locate the ovaries and follicles. Some time extra rectal manipulative assistance may occasionally be needed for better positioning of ovaries and follicles against the probe. The needle guide on the ultrasound screen settings allows the 17-gauge aspiration needle to be aimed correctly and the needle is introduced into a follicle of $10-15 \mathrm{~mm}$ size. The suction is applied by way of the foot control with additional boost vacuum if necessary. The follicular contents are sucked into the collection tube attached to the suction unit. A record was kept of which tubes were used for which ovary so that total number of follicles aspirated could be compared to oocyte recovery. The total volume of fluid from both ovaries was checked by collecting into separate tubes. 


\section{Harvesting of oocytes}

Collection tubes contents were transferred into a square petridish (Falcon 351112, Becton Dickinson USA) to separate the oocytes from the cumulus oocyte complexes manually. Oocytes were teased manually from the complexes using two $20 \mathrm{G}$ X 1.5 inch needles (Monoject USA). Harvested oocytes were counted and prepared for later use either for incubation with sperm or for sperm injection.

\section{Results}

Volume of follicular fluid collected varied greatly and depended on the number and size of follicles present and ease of access to ovary (in case of non surgical). Only ovaries with a minimum of 5 follicles greater than $10 \mathrm{~mm}$ in diameter were attempted. In all cases the minimum amount of fluid collected was $2 \mathrm{ml}$ (from a left ovary with 7 folls of $10-14 \mathrm{~mm}$ dia) to a maximum of $16 \mathrm{ml}$ (left ovary with 8 folls $10-14 \mathrm{~mm}$ dia). Fluid varied from clear through to blood tinged and some collection actually appeared as whole blood.

The number of cumulus complexes and oocytes recovered appears in table 1 . corresponding to:

- Group (A) Nonsurgical collection from both ovaries.

- Group (B) Surgical collection from left ovary only.

- Group (C) Surgical from left and nonsurgical from right.

Table 1. Non-Surgical and Surgical Collection of Oocytes in Camels

\begin{tabular}{cccccc}
\hline \multirow{2}{*}{ Group } & \multirow{2}{*}{ Camel I.D. } & \multicolumn{2}{c}{ Follicle Complex } & \multicolumn{2}{c}{ Oocytes Collect } \\
\cline { 3 - 6 } & & LT. OV. & RT. OV. & LT. OV. & RT.OV \\
\hline & $491^{\mathrm{a}}$ & 20 & 15 & 7 & 6 \\
& $501^{\mathrm{d}}$ & NA & 13 & NA & 6 \\
& 954 & 5 & 7 & 2 & 3 \\
& 4 & 6 & 4 & 1 & 0 \\
Group (A) & 337 & 3 & 4 & 0 & 1 \\
& 154 & 8 & 8 & 6 & 1 \\
Non-Surgical Transvaginal & $603^{\mathrm{b}}$ & 5 & 2 & 4 & 0 \\
Collection of Oocytes & 265 & 7 & 2 & 4 & 2 \\
& 192 & 5 & 1 & 2 & 1 \\
& $105^{\mathrm{C}}$ & 4 & NA & 3 & NA \\
& $953^{\mathrm{d}}$ & & 4 & & 2 \\
& 495 & 5 & 3 & 2 & 1 \\
& 722 & 2 & 2 & 1 & 1 \\
& TOTAL & $\mathbf{7 0}$ & $\mathbf{6 5}$ & $\mathbf{3 2}$ & $\mathbf{2 4}$ \\
\hline \multirow{3}{*}{ Group (B) } & 235 & 4 & & 2 & \\
Surgical Collection of Left & $954^{\mathrm{a}}$ & 7 & & 6 & \\
Ovary Only & 510 & 12 & & 9 & \\
\hline Group (C) & TOTAL & $\mathbf{2 3}$ & & $\mathbf{1 7}$ & \\
\hline Surgical Collection of Left & 261 & 8 & 7 & 6 & 5 \\
Ovary and Transvaginal in & 33 & 8 & 0 & 7 & 0 \\
Right & 294 & 3 & 2 & 1 & 0 \\
\hline & TOTAL & $\mathbf{1 9}$ & $\mathbf{9}$ & $\mathbf{1 4}$ & $\mathbf{5}$ \\
\hline
\end{tabular}


All surgical cases recovered uneventfully and camels were checked ultrasonically for any problems 30 days post collection. In all cases there was no evidence of any excess ovarian bursal fluid and normal follicles were present. A number of the camels were subsequently mated with 3 becoming pregnant on the first attempt.

In the non-surgical collection (13 camels) only the total \% of oocytes recovered per follicles aspirated was $29 \%$. Total \% Oocytes Recovered/Follicles Aspirated $=56 / 191$. The surgical results taken from Group B and C (6 camels) gave a total percentage of $59.6 \%$ for oocytes collected per follicles aspirated. A statistical comparison using Chi-squared for the two techniques showed that the surgical was superior for oocyte recovery with $\mathrm{P}<.0001$. In this experiment if we consider the non-surgical collection was almost exactly half the recovery rate of the surgical then when we factor in that the non-surgical approach allowing simpler access to both ovaries then the actual number of oocytes collected per donor camel is almost equal.

\section{Discussion}

While the surgical recovery was more effective on a per follicle basis it did present many drawbacks when compared to the non-surgical transvaginal method. The most obvious draw back is the time taken to perform the surgery and the fact that only the left side ovary is readily accessible to the flank surgery approach (McKinnon and Tinson unpublished). Surgical aspiration takes around $30 \mathrm{mins}$ for a single ovary compared to 8-10 minutes for both ovaries in the case of transvaginal. Surgery has the added disadvantage of producing adhesion post surgically and thus being a less repeatable procedure. Safe repeatable transvaginal collection was developed as a technique for cattle in hormane stimulated animals in the late 80 's(Pieterse et al.,
1988,1992). Comparing the results to reports in other animals such as cattle the recovery rate and total oocytes is lowering in the camel for nonsurgical. In one report on cattle (Perez et al 1996) the average follicle number aspirated per cow from a group of FSH stimulated cows was 13.2 with a recovery of 8.6 oocytes (65\%). Meintjes et al (1995) reported the \% oocyte recovered per follicle aspirated in nonpregnant cattle as $43 \%$. In our case on group $\mathrm{A}$ the average number of follicles aspirated was 15.7 with the average number of oocytes 4.7. It is difficult to be sure exactly how many follicles are successfully aspirated when hugh number of follicles are present. In table $\mathrm{A}$, there are differences in numbers between the total follicle numbers, the number of follicular complexes (cumulus oocyte complex) and the actual number of oocytes recovered. If the number of complexes is a better measure of the number of follicles actually aspirated then the number for the camel for nonsurgical collection would be much improved. In this case the $\%$ oocytes recovered per cumulus complex recovered was $61 / 132$, which is closer to the quoted figures in cattle. It was noticed by the authors that the separation process of the oocyte from complex in the post collection phase was more difficult than in the human case with the "jelly" being particularly tenacious (Tinson pers obs.). This could possibly cause problems in the camel at the time of collection. The double lumen needles have been tried to attempt flushing the follicles but were not found to be any more efficient than single lumen with suction. Laporoscopic collection is a less invasive technique than the surgical approach of laparotomy and allows easier access to both ovaries. However it still requires more time than the transvaginal technique as well as needing considerable extra investment in equipment. Work in Germany in 1994 (Bungartz et al) also shows that with the recovery rate of 
cumulus oocyte complexes rate of up to $75 \%$ in cattle possible that it is a superior technique to laparoscopic collection. With the past success of the use of sex specific sequences in determining the sex of an embryo (Reed 1986, Herr 1991) and recent success in the camel (Tinson 2001) there is a big opportunity to apply this to the IVF embryo once the technique is further developed in the camel. The advances in twinning are limited (Tinson et al 2001) in comparison to what could be done with blastomere extraction during the early phases of an IVF embryos development. It has been shown with embryo cloning what can be done with cattle (Trounsen pers comm.) and combined with sexing the opportunity for breeding superior milking camels and endangered camels (Hare, 2000) is very good. The timing of the HCG injection to the camel to induce oocyte maturation is an area that is going to need a lot of attention as the technique develops. The camel is an induced ovulator (Novoa 1970) and so if mating doesn't occur or an injection to stimulate ovulation is not given the follicles will continue to grow. So far we have attemped collection of oocytes at $30 \mathrm{hrs}, 24 \mathrm{hrs}$ and 18 hrs post HCG but in all cases we still see the presence of oocytes that are collected with a second polar body seen immediately post collection. This has also been seen in the case of cats, which are also induced ovulators (Trounsen pers comm.). The other problem with the camel appears to be purely anatomical and in some camels the left ovary in particular can be difficult to visualize with the probe making collection difficult with the transvaginal technique.

\section{Conclusion}

In vitro fertilization is an assisted reproductive technique that we have been experimenting with to see the practicality of applying it to camels, particularly in the case of ovarian bursal fluid syndrome, which is often refractory to treatment. It would appear both surgical and non-surgical approaches to the collection of oocytes can give repeatable results in the camel. However the practicalities of the transvaginal method will most likely see more use as our main approach to continuing research into I.V.F. in the camel. The work needed to further develop both I.V.F. and I.C.S.I. (Intra cytoplasmic sperm injection) in the camel will need a further improvement of the collection techniques, but more importantly the assistance of specialists in the laboratory preparation and handling of the oocytes. In human I.V.F. centers the work is split into the specialists for collections and later transfers as well as the specialist technicians for the I.C.S.I and oocyte incubations with a team effort giving success. The whole process is too time consuming and technical to have only a few people follow the process from start to finish.

\section{References}

Carnevale, E.M. and O.J. Ginther, 1993. Use of linear ultrasonic transducer for the transvaginal aspiration and transfer of oocytes in the mare. J. Equine Vet. Sci. 13:25-27.

Bungartz, L., A. Lucas-Hahn, , D. Rath, and H. Nieman. 1995. Collection of Oocytes from Cattle via Follicular Aspirated aided by Ultrasound with or without Gonadotrophin Pretreatment and in Different Reproductive Stages. Theriogenology 43:667-675.

Herr,C., N. Holt, U. Pietrzak, K. Old and K. Reed, 1990. Increased number of pregnancies per collected embryo by bisection of blastocyst stage ovine embryos. Theriogenology 33:244.

Hare J. 2000. Proceedings of the First International Conference on the Wild Two Humped Camel. Beijing pp 2026, August. 
Herr, C. M. and K. C. Reed. 1991. Micromanipulation of bovine embryos for sex determination. Theriogenology $35: 45-54$.

Kuhad K. S., A. H. Tinson, A. Rehman, Rajesh and J. Almasri. 1998. New Technique for Soft Palatectomy in One humped Male Racing Camels.Proceedings of the Third Annual Meeting for Animal Production Under Arid Conditions Vol.2:145-153.

Manefield GW and A. H. Tinson. 1996. "Camels- A Compendium", Vade Mecum SeriesC No 22 Post Grad. Foundation Sydney University. 372 pages.

McKinnon A. O., E. M. Carnevale, E. L. Squires, J. L. Voss and G. E. Seidel. 1988. Heterogenous and xenogenous fertilization of in vivo matured equine oocytes. J. Equine Vet. Sci. 8:143-147.

Mc Kinnon A. O., A. H., Tinson.1992. Embryo Transfer in Dromedary Camels. Proc 1st Int Camel Conf. Eds Allen WR, A. . Higgins, D. Snow and J. F. Wade. Newmarket, Suffolk, UK. R\&W Publications (Newmarket) Ltd., 203-208.

McKinnon A.O., A. H. Tinson and G. Nation. 1994. Embryo Transfer in Dromedary Camels. Theriogenology 41:145-150.

Meintjes M., M. S. Bellow, J. R. Broussard, J. B. Paul and R. A. Godke. 1995. Transvaginal Aspiration of Oocytes from Hormone Treated Pregnant cattle for In Vitro Fertilisation. J. Anim.Sci. 73:967-974.

Novoa C. 1970. Review: Reproduction in Camelidae. J Reprod Fert.; 22:3-20.

Perez O., R. Richard, H. L. Green and Godke. 1996. Transvaginal Oocyte Recovery from Post Partum Beef Cows. Iowa State University.

Pieterse M. C., K. A. Kappen, A. M. Kruip. and M. A. Taverne. 1998. Aspiration of bovine oocytes during Transvaginal Ultrasound Scanning of Ovaries. Theriogenology 30:751.

Pieterse M. C., Vos, A. M. Kruip, Y. A. Wurth, H. Van Beeden, A. H. Willemse, and M. A. Taverne. 1992. Repeated Transvaginal Ultrasound Guided Ovum Pickup in EEG Treated Cows. Theriogenology 37:273.

Reed K. C., M. E. Mathews and M. A. S Jones. 1986. Sex Determination in ruminants using Y-chromosome specific polynucleotides. PCT AU87/00254, WO88/01300.

Tibary A. and A. Anouassi, 1997. Theriogenology in Camelids. Abu Dhabi Publishing pp405-406.

Tinson A. H. , K. Singh and K. S. Kuhad, 1998. Large Scale Management of Camels for Embryo Transfer. International Seminar on Camel Applied Research and Development. N.R.C.C. Bikaner India. August. JCPR.

Tinson A. H., 2001. Production of Pre-sexed Racing Camel Offspring using Embryo Biopsy and Detection of Camelid Sex Specific Polynucleic Acid Sequences.

Tinson, A. H., K. S. Kuhad, K. Singh, R. Sambayal, A. Mugheiry, A. Rahman and J. Al Masri, 2001. Twinning in Camels. 6th Annual Conference for Animal Production under Arid Conditions. Nov. 2001 Al-Ain, UAE. (Abstract).

Wilson R.T. 1984. The Camel. Published Longman Group. Harlo w Essex p 9798. 